\title{
Investigation of Appropriate Inhalation Technique for Mometasone Furoate Dry Powder Inhaler
}

\author{
Haruko Yokoyama, ${ }^{* a}$ Kanako Ito, ${ }^{a}$ Hirokazu Mihashi, ${ }^{b}$ Yasuyuki Shiraishi, ${ }^{b}$ \\ Risa Takayanagi, ${ }^{a}$ and Yasuhiko Yamada ${ }^{a}$ \\ ${ }^{a}$ Department of Clinical Evaluation of Drug Efficacy, School of Pharmacy, Tokyo University of Pharmacy and \\ Life Sciences; 1432-1 Horinouchi, Hachioji, Tokyo 192-0392, Japan: and ${ }^{b}$ Product Development Group R\&D \\ Department, Taisei Kako Co., Ltd.; 2-11-6 Fujinosato, Ibaraki, Osaka 567-0054, Japan. \\ Received April 21, 2015; accepted July 14, 2016
}

\begin{abstract}
The aim of this study was to establish an appropriate inhalation method with a mometasone furoate dry powder inhaler (MF-DPI). Utilizing a tone-based inhalation training device, we investigated the maximum peak inspiratory flow rate time $\left(T_{\max }\right.$ PIFR) and peak inspiratory flow rate (PIFR) to determine whether either had an influence on lung deposition with use of an MF-DPI. A low tone indicated a PIFR of $28 \mathrm{~L} / \mathrm{min}$ and a high tone that of $40 \mathrm{~L} / \mathrm{min}$, while $60 \mathrm{~L} / \mathrm{min}$ was considered to be the standard. We established an inhalation profile in consideration of a human inhalation pattern, in which $T_{\max }$ PIFR was set at $0.5 \mathrm{~s}$ ( $T_{\max }$ PIFR $0.5 \mathrm{~s})$ and $2.5 \mathrm{~s}\left(T_{\max }\right.$ PIFR 2.5 s). The reference cut-off value derived with a cascade impactor test was used for evaluation of the rate of delivered dose in the lung, which was the amount of drug from stage 3 to 7 at all PIFRs. We then investigated the relationship of the fine particle fraction (FPF) with the claimed dose at $T_{\text {max }}$ PIFR of $0.5 \mathrm{~s}$ and PIFR. There were no differences among the $T_{\max }$ PIFR values for the doses emitted from the device or for the rate of delivered doses in stages 3-7. However, FPF for the claimed dose at $40 \mathrm{~L} / \mathrm{min}$ was significantly lower than that at $60 \mathrm{~L} / \mathrm{min}$, which was dependent on PIFR. Our results showed that PIFR but not $\boldsymbol{T}_{\max }$ PIFR has an effect on lung deposition after inhalation with an MF-DPI.
\end{abstract}

Key words mometasone furoate; dry powder inhaler; maximum peak inspiratory flow rate time; peak inspiratory flow rate; lung deposition

A mometasone furoate dry powder inhaler (MF-DPI) is a device utilized for administration of inhaled corticosteroid, which is used for treatment of asthma. MF binds with high affinity to the corticosteroid receptor and has anti-inflammatory potency, ${ }^{1-3)}$ and is formulated for delivery via a breath-actuated DPI using a Twisthaler ${ }^{\circledR}$. Doses emitted from the device have been shown to be high, with flow rates ranging from 28.3 to $\left.70 \mathrm{~L} / \mathrm{min}^{4}{ }^{4}\right)$ That study also revealed that the dose emitted from the device has a relationship with flow rate, ${ }^{4)}$ though the relationship between dose and lung deposition amount is not clear. Furthermore, the mass median aerodynamic diameter (MMAD) of an MF-DPI is $2.0 \mu \mathrm{m}$, the smallest among available, thus it is characterized by the design of the particles used, which reach from the central to small airway. An inhalation training device (manufactured by Taisei Kako Co., Ltd.) that emits tones and is used in a manner similar to an MF-DPI has been produced to practice inhalation. This device produces a low tone at a peak inspiratory flow rate (PIFR) of $28 \mathrm{~L} /$ min and a high tone at that of $40 \mathrm{~L} / \mathrm{min}$. However, the relationship between PIFR-produced tones and the dose delivered to the lung has not been examined.

The rate of drug deposition in the lung has been examined in vitro in inhalation experiments. Those results revealed that PIFR actually sensed by patients was not reflected in those experimental results, as resistance to inhalation was not included in measured PIFR. In addition, individualized inhalation profiles can not be reproduced, because a vacuum pump is generally used for inhaling the drug in an in vitro setting. Hence, only PIFR values have been examined.

The time to reach maximum PIFR ( $T_{\max }$ PIFR) is extended in patients with respiratory dysfunction, such as chronic ob- structive pulmonary disease and asthma. ${ }^{5,6)}$ Thus, data regarding the rate of drug deposition in the lung in consideration of that influence is required for clinical application, though has not been reported. We previously developed an inhalation simulator that can reproduce a rising inspiratory flow rate similar to a human inhalation pattern.

The aim of the present study was to establish an appropriate inhalation method for MF-DPI using a training device. We investigated whether $T_{\max }$ PIFR or PIFR, based on the tone produced by the training device, had an influence on lung deposition when using an MF-DPI. In addition, the rate of the delivered dose in the throat with a cascade impactor was evaluated, as well as that in the oral cavity and pharynx.

\section{MATERIALS AND METHODS}

Calculation of Rate of Drug Deposition after Inhalation under Various Conditions We used an Asmanex ${ }^{\circledR}$ Twisthaler $^{\circledR} 100 \mu \mathrm{g}$ (MSD K.K., Tokyo, Japan) as the MF-DPI in the present study. In addition, we employed our previously developed inhalation simulator, which consists of a flow recorder placed in an air-tight box covering the MF-DPI and equipped with a cascade impactor, flow control system, and vacuum pump (Fig. 1). Flow rate in the monitor was recorded using the spirometer. The flow control system was installed with a small flow control valve and the inspiratory flow rate was controlled by a computer. The flow rate was increased stepwise using a computer program and inspiration time, $T_{\max }$ PIFR, and PIFR were also controlled. However, PIFR was not decreased stepwise, because our aim was to elucidate the influence of $T_{\max }$ PIFR on lung deposition. Thus, we developed 


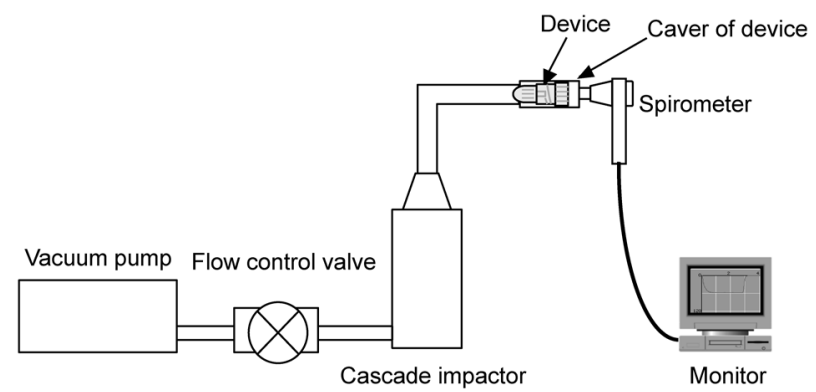

Fig. 1. Schematic Diagram Showing Apparatus Employed to Measure Dose Obtained with Cascade Impactor and Inspiratory Flow Rate

\section{A) $T_{\max }$ PIFR $0.5 \mathrm{~s}$}

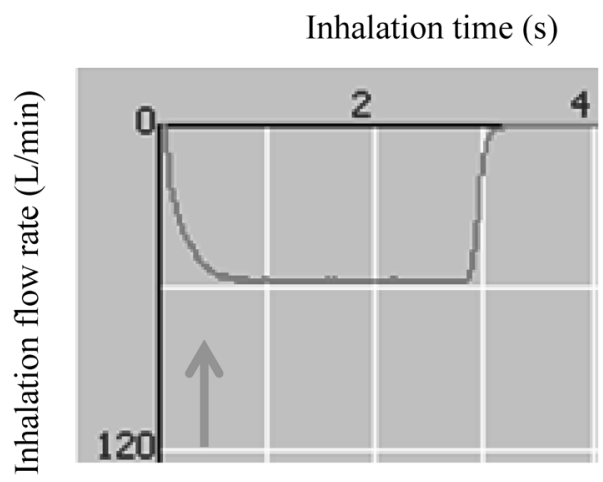

Fig. 2. Inhalation Profiles

$$
\text { (A) } T_{\max } \text { PIFR } 0.5 \mathrm{~s} \text {. (B) } T_{\max } \text { PIFR } 2.5 \mathrm{~s} \text {. }
$$

the present flow control system to reproduce the inhalation profile and did not modify the cascade impactor. The doses delivered to the throat and at each stage were collected using a $50-\mathrm{mL}$ mixture of methanol-water-acetonitrile $(10: 7: 3)$ after a single inhalation from the MF-DPI at a dose of $100 \mu \mathrm{g}$ for $3 \mathrm{~s}$. MF doses in the collected samples were measured using high performance liquid chromatography. We considered that the $T_{\max }$ PIFR value for patients with stable mild asthma was $0.5 \mathrm{~s}\left(T_{\max }\right.$ PIFR $\left.0.5 \mathrm{~s}\right)$, while that of those with stable severe asthma was considered to be $2.5 \mathrm{~s}$ ( $T_{\max }$ PIFR $2.5 \mathrm{~s}$ ) (Fig. 2). PIFR at $28 \mathrm{~L} / \mathrm{min}$ resulted in sounding of a low tone from the training device and $40 \mathrm{~L} / \mathrm{min}$ sounded a high tone, while $60 \mathrm{~L} /$ min was considered to be the standard.

Influence of $\boldsymbol{T}_{\max }$ PIFR on Rate of Delivered Dose into Lung In general, a fine particle fraction (FPF) size of $<5.0 \mu \mathrm{m}$ was considered to be likely to reach the lung (from central to small airway). The cut-off diameters for each stage were calculated by substitution of PIFR in the relational equation at various PIFR values. ${ }^{7)}$ However, it is uncertain whether this equation is reliable, because there is no evidence for a $T_{\max }$ PIFR value of $2.5 \mathrm{~s}$. Therefore, the reference cut-off value in the cascade impactor test was used for evaluation of the rate of the doses delivered into the lung and the amount of drug in stages 3 to 7 was assessed at each PIFR. In addition, we used a PIFR of $28.3 \mathrm{~L} / \mathrm{min}$ for $5 \mathrm{~s}$, which is the reference cascade impactor test.

We calculated the actual emitted dose from the device as a percentage of the label claim, the rate of delivered dose in stages 3-7 for the claimed dose, and the rate of delivered dose
Table 1. Values for Each Cascade Impactor Stage at Flow Rates of 28, 40 , and $60 \mathrm{~L} / \mathrm{min}$

\begin{tabular}{cccc}
\hline \hline \multirow{2}{*}{ Stage } & \multicolumn{3}{c}{ Cut-off value $(\mu \mathrm{m})$} \\
\cline { 2 - 4 } & $28 \mathrm{~L} / \mathrm{min}$ & $40 \mathrm{~L} / \mathrm{min}$ & $60 \mathrm{~L} / \mathrm{min}$ \\
\hline 0 & $>11.06$ & $>9.25$ & $>7.56$ \\
1 & $7.04-11.05$ & $5.89-9.25$ & $4.81-7.56$ \\
2 & $4.73-7.03$ & $3.95-5.88$ & $3.23-4.80$ \\
3 & $3.32-4.72$ & $2.78-3.94$ & $2.27-3.22$ \\
4 & $2.11-3.31$ & $1.77-2.77$ & $1.44-2.26$ \\
5 & $1.11-2.10$ & $0.93-1.76$ & $0.76-1.43$ \\
6 & $0.65-1.10$ & $0.55-0.92$ & $0.45-0.75$ \\
7 & $0.43-0.64$ & $0.36-0.54$ & $0.30-0.44$ \\
\hline
\end{tabular}

B) $T_{\max }$ PIFR $2.5 \mathrm{~s}$

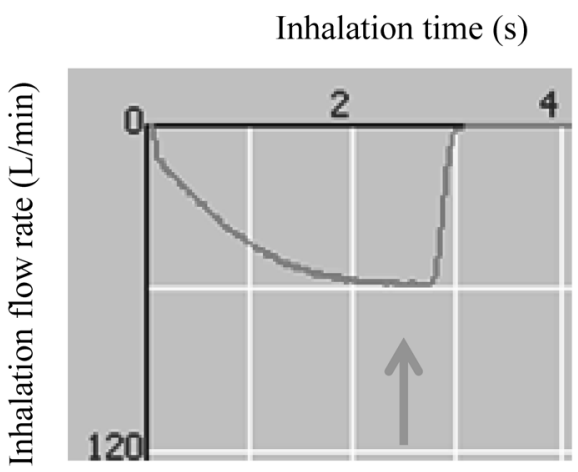

in stages 3-7 for the emitted dose using the apparatus and conditions described above. We compared between the rate of the delivered dose in stages $3-7$ at $T_{\max }$ PIFR values of $0.5 \mathrm{~s}$ and $2.5 \mathrm{~s}$ at PIFRs of 28,40 , and $60 \mathrm{~L} / \mathrm{min}$. Significance was determined using an unpaired $t$-test when comparing 2 groups with the software package JMP ${ }^{\circledR} 8.0$ (SAS, Inc., Tokyo, Japan). A value of $p<0.05$ was considered to be significant.

Influence of PIFR on Rate of Dose into Lung We compared between the FPF of PIFR of 28,40 , and $60 \mathrm{~L} / \mathrm{min}$ at a $T_{\max }$ PIFR value of $0.5 \mathrm{~s}$, which was considered to have scant influence on the cut-off value, because a $T_{\max }$ PIFR of $0.5 \mathrm{~s}$ is a short amount of time to reach PIFR. The cut-off diameters for each stage were calculated by substitution of PIFR in the equation. ${ }^{7)}$ The calculated cut-off values for each stage are shown in Table 1. The cumulative amount of drug in stages 3-7 was assessed by the cut-off value for FPF $(<5.0 \mu \mathrm{m})$ at $28 \mathrm{~L} / \mathrm{min}$ and $40 \mathrm{~L} / \mathrm{min}$, while that in stages $2-7$ was assessed for FPF $(<5.0 \mu \mathrm{m})$ at $60 \mathrm{~L} / \mathrm{min}$. Significance was determined using Tukey's honestly significant difference test for multiple comparisons with the software package JMP ${ }^{\circledR} 8.0$ (SAS, Inc., Tokyo, Japan). A value of $p<0.05$ was considered to be significant.

\section{RESULTS}

Influence of $T_{\max }$ PIFR on Rate of Delivered Dose into Lung The rates of delivered dose at each stage with a $T_{\max }$ PIFR of $0.5 \mathrm{~s}$ and $2.5 \mathrm{~s}$ at 28,40 , and $60 \mathrm{~L} / \mathrm{min}$ are shown in Table 2. In addition, those at the reference PIFR of $28.3 \mathrm{~L} / \mathrm{min}$ 
for $5 \mathrm{~s}$ are shown in the right column. Furthermore, the dose emitted from the device as a percentage of label claim, the rate of the delivered dose in stages 3-7 for the claimed dose, and the rate of the delivered dose in stages 3-7 for the emitted dose at a $T_{\max }$ PIFR of 0.5 and $2.5 \mathrm{~s}$ are shown in Fig. 3. For each emitted dose, the delivered doses in stages 3-7 for the claimed and the emitted doses were not different between $T_{\max }$ PIFR 0.5 and $2.5 \mathrm{~s}$. Moreover, there were no differences for the rate of delivered dose in stages 3-7 for the claimed doses, while there was a significant difference for the rate of emitted dose from the device between the reference cascade impactor test (at a PIFR of $28.3 \mathrm{~L} / \mathrm{min}$ for $5 \mathrm{~s}$ ) and a PIFR of $28 \mathrm{~L} / \mathrm{min}$ for $3 \mathrm{~s}$.

Influence of PIFR on Rate of Delivered Dose into Lung FPF values for the claimed doses with PIFRs at 28, 40, and $60 \mathrm{~L} / \mathrm{min}$ at a $T_{\max }$ PIFR of $0.5 \mathrm{~s}$ are shown in Fig. 4. Those at $60 \mathrm{~L} / \mathrm{min}$ were significantly higher than at $40 \mathrm{~L} / \mathrm{min}(p=0.03)$, while there were no differences between the values obtained at 28 and $40 \mathrm{~L} / \mathrm{min}(p=0.93)$, or between those obtained at 28 and $60 \mathrm{~L} / \mathrm{min}(p=0.06)$.

\section{DISCUSSION}

Drug delivery performance is an index used for evaluating DPI formulations. In human trials, a radiolabeled drug is generally administered by subject inhalation, though there are concerns regarding safety. On the other hand, human inspiration patterns can not be reproduced in vitro, as inhalation is performed by a vacuum pump in laboratory conditions. We previously developed an in vitro experimental device that is able to control $T_{\max }$ PIFR. It has been reported that most of the dose is emitted from a DPI device before reaching $T_{\max }$ PIFR, ${ }^{8}$ thus indicating that drug deposition in the lungs might be affected by that value, though no findings have been reported to confirm that speculation. On the other hand, related studies have been presented. A relationship between $T_{\max }$ PIFR and rate of carrier particle size released from the Rotahaler was shown. ${ }^{9}$ However, that study only measured the carrier particles released from the device, thus the results did not clearly reveal the relationship between $T_{\max }$ PIFR and FPF. Moreover, another study measured FPF $(\%<5 \mu \mathrm{m})$ using laser diffraction and found it to be related with $T_{\max }$ PIFR. ${ }^{10)}$ Those findings showed that terbutaline FPF was dependent upon a $T_{\max }$ PIFR of $60 \mathrm{~L} / \mathrm{min}$, when $T_{\max }$ PIFR was in a range from 0.1 to $0.3 \mathrm{~s}$, whereas there was no relationship with $T_{\max }$ PIFR ranging from 0.3 to $0.5 \mathrm{~s}$. The $T_{\max }$ PIFR of examined patients was reported to be greater than $0.5 \mathrm{~s},{ }^{11)}$ though that was not examined in that previous study. In addition, we speculated that $T_{\max }$ PIFR would gradually shorten from the maximum at $2.5 \mathrm{~s}$, though found no difference for the rate of delivered doses in stages $3-7$ between 0.5 and $2.5 \mathrm{~s}$. Consequently, we examined it only at 0.5 and $2.5 \mathrm{~s}$.

The relationships between various inhalation flow profiles and drug dispersion have been examined, ${ }^{12)}$ with high, sharp drug releasing pattern shown when $T_{\max }$ PIFR was short. When $T_{\max }$ PIFR was long, the drug releasing pattern had several peaks and was lower than that as compared to when $T_{\max }$ PIFR was short. It was concluded that the inhalation flow profile and drug releasing pattern are related. Therefore, it is possible that the sum of the released drug and drug delivery in the lungs is the same when PIFR is the same and $T_{\max }$ PIFR is different. In the future, it will be important to examine various factors that affect drug delivery into the lungs.

It is known that the PIFR of each patient has effects on drug delivery with a $\mathrm{DPI}^{5,6,11,13)}$ and each pharmaceutical company has prepared their own DPI training device for confirming inspiratory flow rate. However, no previous study examined the relationship between PIFR of the training device and drug deposition in the lungs. In the present study, we investigated the effect of $T_{\max }$ PIFR on drug deposition in the lungs at PIFR noted by a tone produced when using an MFDPI training device.

As the result of future research, we hope to develop a cascade impactor without the need for a pre-separator. For doses of 200 and $400 \mu \mathrm{g}$, the MF-DPI delivered 40.8 and $36.7 \%$, respectively, of the claimed delivered doses with particles sized $<8.6 \mu \mathrm{m}$ (stages $1-7$ ) at $60 \mathrm{~L} / \mathrm{min}^{4}$.) In the present study, $100 \mu \mathrm{g}$ delivered at a $T_{\max }$ PIFR of 0.5 and $2.5 \mathrm{~s}$ resulted in

Table 2. Rate of Delivered Dose in Each Stage of Cascade Impactor with $T_{\max }$ PIFR of 0.5 and $2.5 \mathrm{~s}$ at $28,40,60 \mathrm{~L} / \mathrm{min}$, and $28.3 \mathrm{~L} / \mathrm{min}$ (Reference)

\begin{tabular}{|c|c|c|c|c|c|c|c|}
\hline \multirow{2}{*}{$\frac{\text { PIFR }}{T_{\max } \text { PIFR }}$} & \multicolumn{2}{|c|}{$28 \mathrm{~L} / \mathrm{min}$} & \multicolumn{2}{|c|}{$40 \mathrm{~L} / \mathrm{min}$} & \multicolumn{2}{|c|}{$60 \mathrm{~L} / \mathrm{min}$} & \multirow{2}{*}{$\frac{28.3 \mathrm{~L} / \mathrm{min}}{-}$} \\
\hline & $0.5 \mathrm{~s}$ & $2.5 \mathrm{~s}$ & $0.5 \mathrm{~s}$ & $2.5 \mathrm{~s}$ & $0.5 \mathrm{~s}$ & $2.5 \mathrm{~s}$ & \\
\hline \multicolumn{8}{|c|}{ Rate of delivered dose $(\%)$} \\
\hline Throat & $8.7(2.0)$ & $10.4(4.8)$ & $9.1(1.6)$ & $11.4(8.6)$ & $11.4(2.4)$ & $15.2(10.3)$ & $5.4(1.5)$ \\
\hline 0 & $10.5(6.9)$ & $8.2(5.0)$ & $8.3(1.3)$ & $7.7(2.6)$ & $11.9(4.0)$ & $6.5(3.1)$ & $7.0(1.9)$ \\
\hline 1 & $19.4(5.8)$ & $12.7(4.1)$ & $15.4(4.5)$ & $12.8(5.5)$ & $9.3(1.5)$ & $13.4(7.2)$ & $11.7(1.7)$ \\
\hline 2 & $11.2(3.9)$ & $8.1(7.6)$ & $11.3(3.0)$ & $10.9(5.8)$ & $9.9(4.3)$ & $5.8(2.2)$ & $7.0(2.4)$ \\
\hline 3 & $9.9(6.2)$ & $3.9(2.6)$ & $5.2(1.8)$ & $5.0(3.9)$ & $4.4(2.2)$ & $2.9(1.0)$ & $4.7(2.0)$ \\
\hline 4 & $3.9(1.9)$ & $3.7(1.8)$ & $3.5(1.2)$ & $3.8(1.6)$ & $6.2(3.4)$ & $10.4(5.7)$ & $2.0(1.0)$ \\
\hline 5 & $3.3(2.2)$ & $3.6(2.6)$ & $5.9(2.0)$ & $5.6(2.9)$ & $5.2(1.9)$ & $7.5(3.2)$ & $4.2(2.9)$ \\
\hline 6 & $3.9(1.8)$ & $3.4(2.2)$ & $5.4(1.2)$ & $4.4(1.7)$ & $6.2(1.8)$ & $6.1(2.0)$ & $3.0(0.4)$ \\
\hline 7 & $2.4(1.0)$ & $4.0(3.2)$ & $1.5(0.5)$ & $2.0(1.4)$ & $4.3(1.3)$ & $3.5(1.6)$ & $2.4(0.2)$ \\
\hline Rate of dose released form device (\%) & $73.2(16.0)$ & $57.8(11.4)$ & $65.7(5.6)$ & $63.6(10.3)$ & $68.8(12.0)$ & $71.4(14.4)$ & $47.4(2.7)$ \\
\hline $\begin{array}{l}\text { Rate of dose delivered in stages } 3-7 \text { for } \\
\text { claimed dose }(\%)\end{array}$ & $23.4(8.4)$ & $18.5(4.5)$ & 21.5 & $20.9(8.7)$ & $26.4(9.5)$ & $30.4(9.3)$ & 16.3 \\
\hline FPF (\%) & $23.4(8.4)$ & - & 21.5 & - & $36.3(10.5)$ & - & 16.3 \\
\hline
\end{tabular}

$n=5$, mean (S.D.), FPF; fine particle fraction. 

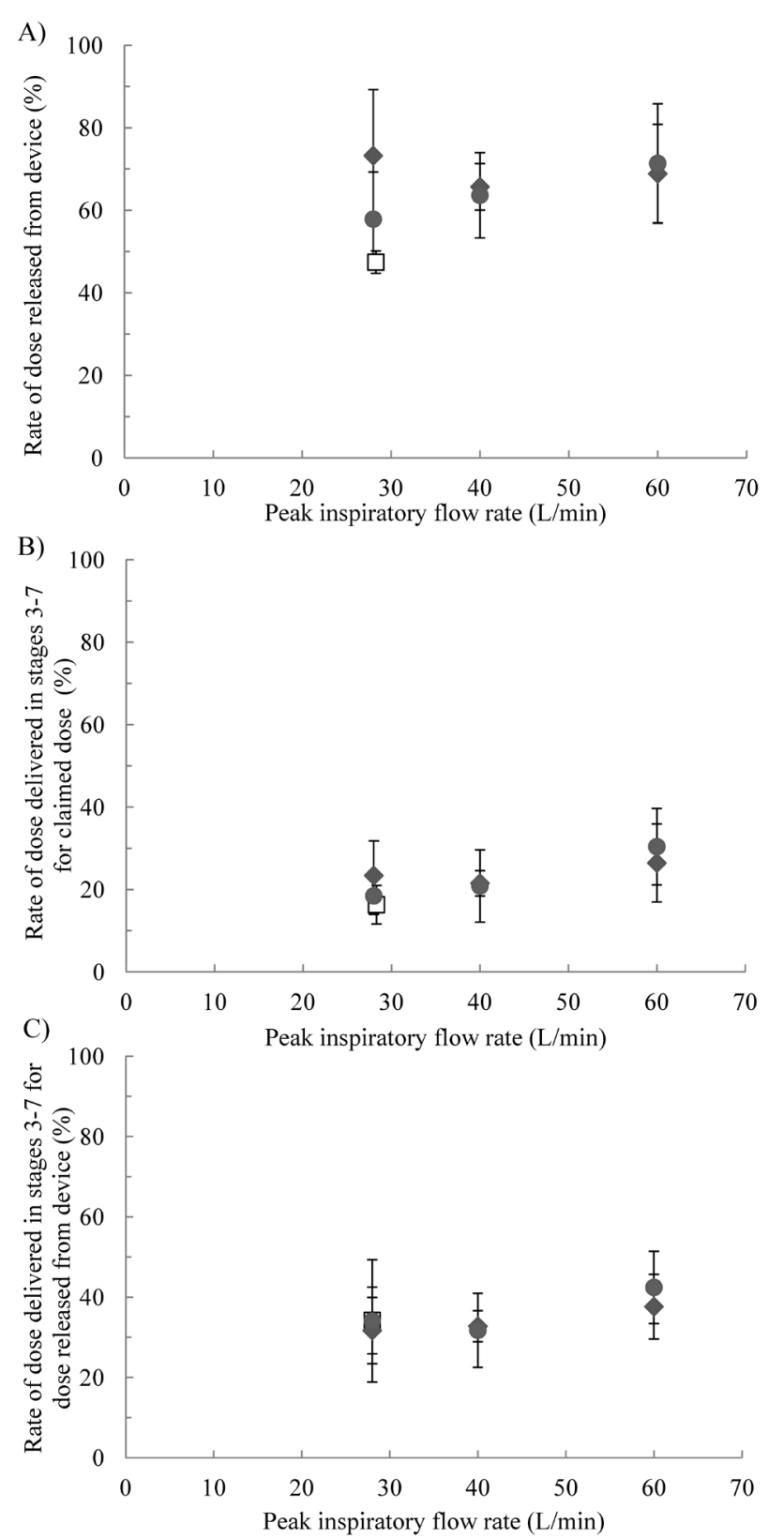

Fig. 3. Comparisons of $T_{\max }$ PIFR Values at 0.5 and $2.5 \mathrm{~s}$ for (A) Rate of Dose Released from Device, (B) Rate of Dose Delivered in Stages 3-7 for Claimed Dose, and (C) Rate of Dose Delivered in Stages 3-7 for Dose Released from Device at Each Tested Peak Inspiratory Flow Rate $(n=5)$

४: $T_{\max }$ PIFR $0.5 \mathrm{~s}, \bullet: T_{\max }$ PIFR $2.5 \mathrm{~s}, \square:$ Reference cascade impactor test $(28.3 \mathrm{~L} / \mathrm{min}$ for $5 \mathrm{~s})$.

45.6 and $49.6 \%$, respectively, of the claimed delivered dose in stages $1-7$ at $60 \mathrm{~L} / \mathrm{min}$. Thus, we think that the pre-separator is not necessary, as the results were quite similar.

The $T_{\max }$ PIFR of asthma patients in a stable phase has been reported to be $0.5 \mathrm{~s} .{ }^{11)}$ Thus, we used $T_{\max }$ PIFRs of 0.5 and $2.5 \mathrm{~s}$ as maximal values in the present study. Several studies set the inspiration time at $2 \mathrm{~s}$ by taking vital capacity into consideration, as the time required for a patient to strongly inhale with the DPI is approximately $2 \mathrm{~s}^{4,14)}$ On the other hand, it has been noted that the dose emitted from a DPI is not affected by inspiration time. ${ }^{8)}$ Moreover, the average lung capacity of Japanese healthy adults is $3.5 \mathrm{~L}$ in males and $2.5 \mathrm{~L}$ in females. Thus, a $T_{\max }$ PIFR of $2.5 \mathrm{~s}$ was set as the maximal value in

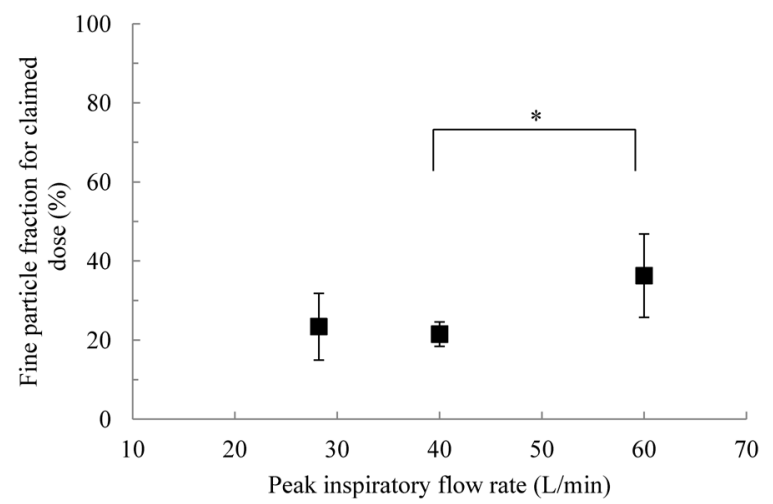

Fig. 4. Relationships of Peak Inspiratory Flow Rate with Each Value and Fine Particle Fraction (FPF) Value for Claimed Dose at a $T_{\max }$ PIFR of $0.5 \mathrm{~s}(n=5)$

the present study, while total inspiration time was set at $3 \mathrm{~s}$. A PIFR of $28 \mathrm{~L} / \mathrm{min}$ with the training device is equivalent to the maximum flow rate of a 3-year-old child with asthma, while that of $60 \mathrm{~L} / \mathrm{min}$ is the same as that of an 8 -year-old child with asthma. ${ }^{15)}$ It is considered impossible for children younger than 3 years old to use a DPI. Accordingly, a PIFR of $28 \mathrm{~L} / \mathrm{min}$ is considered reasonable for nearly all patients. In addition, we found no differences in the rate of delivered dose in stages 3-7 for the claimed dose with the reference cascade impactor at a PIFR of $28.3 \mathrm{~L} / \mathrm{min}$ for $5 \mathrm{~s}$ as compared to that at $28 \mathrm{~L} / \mathrm{min}$ for $3 \mathrm{~s}$. However, the dose released from the device was significantly different between the reference cascade impactor test and each group, except for a PIFR of $28 \mathrm{~L} / \mathrm{min}$ with a $T_{\max }$ PIFR of $2.5 \mathrm{~s}$. Also, the rate of the delivered dose in the throat by the reference cascade impactor test was lower as compared to the others, though the reason is unclear.

Since the emitted dose and rate of the delivered dose in stages 3-7 for the claimed dose, and that for the emitted dose from the device did not vary in regard to $T_{\max }$ PIFR, it is suggested that drug delivery in the lungs by the MF-DPI might not be affected by $T_{\max }$ PIFR. Thus, we concluded that PIFR exerts effects on drug delivery by an MF-DPI without dependence on inhalation pattern.

As for the effect of PIFR on drug delivery into the lungs, we considered that the doses emitted from the device are not affected by a PIFR in the range of $28-60 \mathrm{~L} / \mathrm{min}$ and our findings were consistent with others previously reported. ${ }^{4)}$ Meanwhile, the cut-off diameters were useful to calculate with the relational equation ${ }^{7)}$ at a $T_{\max }$ PIFR of $0.5 \mathrm{~s}$. It was also suggested that FPF for the claimed dose was significantly lower at a flow rate of $40 \mathrm{~L} / \mathrm{min}$ as compared to $60 \mathrm{~L} / \mathrm{min}$, and also tended to be lower at a flow rate of $28 \mathrm{~L} / \mathrm{min}$ as compared to that of $60 \mathrm{~L} / \mathrm{min}(p=0.06)$. Thus, PIFR was shown to affect drug deposition in the lung after inhalation with the MF-DPI.

Our results indicate that drug delivery into the lungs by the present MF-DPI was not affected by $T_{\max }$ PIFR, but rather by PIFR. Furthermore, they confirmed that the MF-DPI is capable to emit a sufficient dose even at a low PIFR of $28 \mathrm{~L} /$ min, which produced a tone by the training device. In future studies, it will be necessary to clarify the mechanisms related to our results.

Acknowledgment We thank Dr. Tetsuri Kondo for the valuable comments. 
Conflict of Interest Yasuyuki Shiraishi is Executive Director of Taisei Kako Co., Ltd. Hirokazu Mihashi is an employee of Taisei Kako Co., Ltd. The other authors have no conflict of interest to declare. A training device is manufactured by Taisei Kako Co., Ltd.

\section{REFERENCES}

1) Bousquet J. Mometasone furoate: an effective anti-inflammatory with a well-defined safety and tolerability profile in the treatment of asthma. Int. J. Clin. Pract., 63, 806-819 (2009).

2) Johnson M. Development of fluticasone propionate and comparison with other inhaled corticosteroids. J. Allergy Clin. Immunol., 101, S434-S439 (1998).

3) Valotis A, Högger P, Neukam K, Elert O, Human receptor kinetics, tissue binding affinity, and stability of mometasone furoate. $J$. Pharm. Sci., 93, 1337-1350 (2004).

4) Yang TT, Li S, Wyka B, Kenyon D. Drug delivery performance of the mometasone furoate dry powder inhaler. J. Aerosol Med., 14, 487-494 (2001).

5) de Boer AH, Winter HMI, Lerk CF. HMIW, Lerk CF. Inhalation characteristics and their effects on in vitro drug delivery from dry powder inhalers Part 1. Inhalation characteristics, work of breathing and volunteers' preference in dependence of the inhaler resistance. Int. J. Pharm., 130, 231-244 (1996).

6) Burnell PK, Small T, Doig S, Johal B, Jenkins R, Gibson GJ. Exvivo product performance of Diskus and Turbuhaler inhalers using inhalation profiles from patients with severe chronic obstructive pulmonary disease. Respir. Med., 95, 324-330 (2001).

7) Taki M, Marriott C, Zeng XM, Martin GP. Aerodynamic deposition of combination dry powder inhaler formulations in vitro: a comparison of three impactors. Int. J. Pharm., 388, 40-51 (2010).

8) Bisgaard H, Klug B, Sumby BS, Burnell PK. Fine particle mass from the Diskus inhaler and Turbuhaler inhaler in children with asthma. Eur. Respir. J., 11, 1111-1115 (1998).

9) Chavan V, Dalby R. Effect of rise in simulated inspiratory flow rate and carrier particle size on powder emptying from dry powder inhalers. AAPS PharmSci, 2, E10 (2000).

10) Martin GP, Marriott C, Zeng XM. Influence of realistic inspiratory flow profiles on fine particle fractions of dry powder aerosol formulations. Pharm. Res., 24, 361-369 (2007).

11) Tarsin WY, Pearson SB, Assi KH, Chrystyn H. Emitted dose estimates from Seretide Diskus and Symbicort Turbuhaler following inhalation by severe asthmatics. Int. J. Pharm., 316, 131-137 (2006).

12) Kondo T, Hibino M, Ohe M, Akazawa K, Tanigaki T, Kato S. Feasibility of instruction of dry powder inhaler usage showing inhalation flow profile and drug dispersion. The Journal of the Japan Society for Respiratory Care and Rehabilitation, 25, 222-224 (2015).

13) Selroos O, Borgström L, Ingelf J. Performance of turbuhaler((R)) in patients with acute airway obstruction and COPD, and in children with asthma: understanding the clinical importance of adequate peak inspiratory flow, high lung deposition, and low in vivo dose variability. Treat. Respir. Med., 5, 305-315 (2006).

14) Yokoyama H, Yamamura Y, Ozeki T, Iga T, Yamada Y. Analysis of relationship between peak inspiratory flow rate and amount of drug delivered to lungs following inhalation of fluticasone propionate with a Diskhaler. Biol. Pharm. Bull., 30, 162-164 (2007).

15) Amirav I, Newhouse MT, Mansour Y. Measurement of peak inspiratory flow with in-check dial device to simulate low-resistance (Diskus) and high-resistance (Turbohaler) dry powder inhalers in children with asthma. Pediatr. Pulmonol., 39, 447-451 (2005). 